\title{
Archéopages
}

Archéopages

Archéologie et société

42 | 04-07/2015

Construire en terre crue

\section{Terre et peinture à l'époque romaine}

Pratiques architecturales et décoratives en Gaule romaine mises en évidence par l'analyse toichographologique

Earth and paint in the Roman period. Architectural and decorative practices in Roman Gaul revealed by the analysis of painted plaster

Barro y pintura en la época romana. Prácticas arquitectónicas y decorativas en la Galia romana reveladas gracias al análisis de grafología mural

\section{Julien Boislève}

\section{OpenEdition}

\section{Journals}

Édition électronique

URL : https://journals.openedition.org/archeopages/1185

DOI : $10.4000 /$ archeopages. 1185

ISSN : 2269-9872

\section{Éditeur}

INRAP - Institut national de recherches archéologiques préventives

Édition imprimée

Date de publication : 1 mars 2016

Pagination : 48-57

ISSN : 1622-8545

Référence électronique

Julien Boislève, "Terre et peinture à l'époque romaine », Archéopages [En ligne], 42 | 04-07/2015, mis en ligne le 01 juillet 2017, consulté le 02 juin 2021. URL : http://journals.openedition.org/archeopages/ 1185 ; DOI : https://doi.org/10.4000/archeopages.1185 


\section{Terre et peinture à l'époque romaine Pratiques architecturales et décoratives en Gaule romaine mises en évidence par l'analyse toichographologique}

Julien Boislève Irrap, UMR R546, «AOROC,

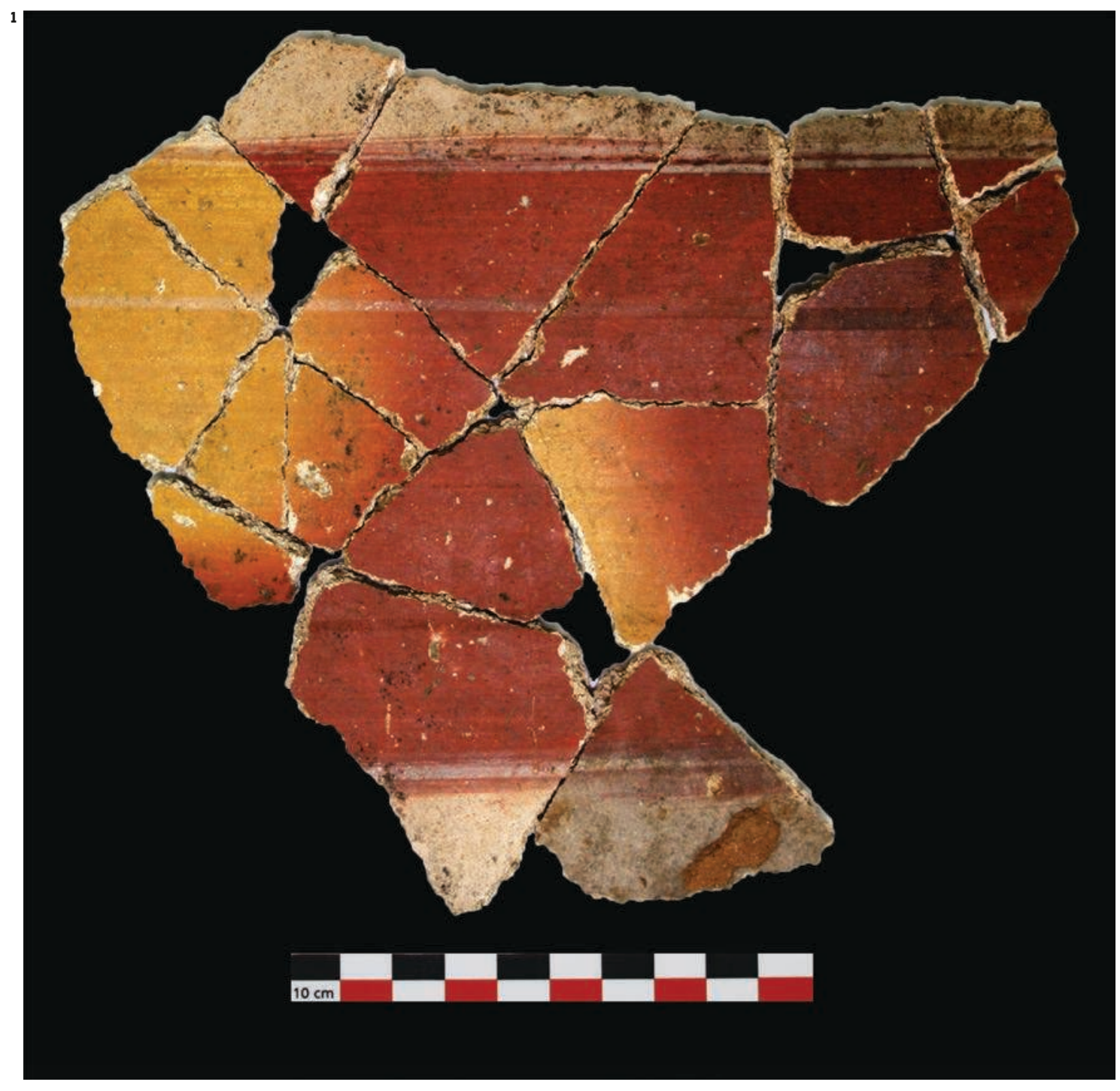

1. L'ocre jaune, chargée de goethite, subit sous

l'action du feu une

modification de teinte qui

la transforme en ocre

rouge.Vitruve mentionne

clairement cette pratique

pour la production de terre

rouge. On l'observe aussi

de manière accidentelle,

comme ici à Arles, lorsque

les flammes d'un incendie

ont léché la paroi peinte

en jaune. 
Appréhender l'usage architectural du matériau de construction que constitue la terre sous l'angle de l'enduit mural d'époque romaine peut sembler une gageure tant la technique même du décor à fresque découle de la mise en œuvre de la chaux sous la forme de mortier. Néanmoins, l'évolution des méthodes de fouille et d'enregistrement des données, parallèlement à celle des analyses, et l'attention croissante portée aux enduits peints et à leur étude permettent un dialogue plus précis entre sources antiques, vestiges d'architectures en terre abordés par l'archéologie, restitution des élévations et indices des modes de construction enregistrés par l'étude toichographologique. Concernant l'usage de la terre en lien avec le décor peint, trois aspects peuvent être ici approchés : l'emploi même de ce matériau comme peinture, celui mis en évidence dans la réalisation de son support, c'est-àdire l'enduit et ses techniques de pose, et, de manière plus indirecte, l'architecture de terre révélée par l'étude de ce mobilier archéologique, notamment par l'observation des revers.

\section{La terre comme pigment}

Le mot même de terre reste relativement imprécis du point de vue géologique, désignant de manière large une formation meuble à granulométrie fine (Foucault, Raoult, 2005). De par leurs composants constitutifs, ces sédiments présentent une vaste gamme de coloration, le plus souvent naturelle, qui a très tôt intéressé l'art et l'artisanat décoratif pour la réalisation de peinture. La terre, ou plutôt les terres, fournissent donc une bonne part des pigments employés à fresque sur les peintures murales de l'époque romaine, d'autant que les minéraux, comme le souligne déjà Pline dans son Histoire Naturelle, résistent beaucoup mieux à ce qu'il appelle l'amertume de la chaux qui corrompt toutes les couleurs végétales, et présentent donc un avantage technique en plus de l'intérêt de leur coloration.

Les textes antiques, notamment ceux de Vitruve et de Pline, constituent justement une source non négligeable sur l'emploi, la provenance, l'extraction et la mise en œuvre de ces terres colorantes. Ils ont été maintes fois commentés mais demeurent une documentation assez précise qui peut désormais être plus facilement mise en parallèle avec les découvertes archéologiques, à la faveur notamment du développement des analyses. Ainsi, les ocres,

1. Méthode d'analyse non destructive basée sur la mesure de la fréquence d'un rayonnement lumineux. Elle permet la caractérisation de la composition moléculaire d'un matériau.

2. Fouille menée en 2006-2007 sous la direction de J.-Y. Breuil, Inrap, analyse $S$. Pagès, C2RMF.

3. Un quatrième décor, où la couleur est plus sombre, mériterait une détermination par analyse. principalement) sont les plus largement employées en peinture du fait de leur évidente abondance et de leur facilité d'accès [ill. 1]. Les gisements sont en effet nombreux, y compris en Gaule où les carrières du Roussillon, près d'Apt, sont par exemple déjà exploitées. Toutefois, les auteurs latins rappellent qu'il existe bien des qualités différentes et que leur coût varie considérablement. Ainsi, le sil, identifié par la plupart des traducteurs comme de locre jaune, est vendu deux deniers la livre s'il vient d'Attique et seulement un peu plus de deux sesterces s'il s'agit de celui extrait en Gaule (Augusti, 1967; Barbet 1990). Ces mentions traduisent un véritable commerce des pigments et une réputation de certains gisements pour la qualité de matériau qu'ils fournissent. Certains sont même recherchés comme palliatif économique des pigments de très haute valeur comme le cinabre, un minerai rare et qui nécessite une transformation complexe. Pline (Pline l'Ancien, 2002, XXXV, 14) mentionne ainsi la terre de Lemnos dont la couleur approche du vermillon. Si elle est moins coûteuse, elle est tout de même vendue cachetée et sert également à falsifier le cinabre pour en diminuer le coût.

Les terres vertes sont assez révélatrices de ce commerce, parfois lointain et illustré tant par les sources littéraires que par les témoignages archéologiques. Elles appartiennent à la famille des argiles avec deux variantes principales, selon qu'il s'agit de glauconies ou de céladonite. Si les premières sont assez facilement accessibles en Gaule, la seconde est toujours d'importation avec deux zones géographiques d'extraction identifiées par analyse, à Chypre dans le massif de Troodos et, en Italie, soit dans le Monte-Baldo près de Vérone, soit dans le Val di Fassa (Delamare, 1987). La spectrométrie Raman ${ }^{1}$ permet souvent d'établir non seulement la nature de ces pigments mais également leur origine. La céladonite n'apparaît à ce jour que sur un nombre restreint de sites où elle accompagne généralement des décors de haute qualité au sein d'habitats luxueux. La relative rareté du pigment et son origine lointaine en font un matériau coûteux réservé à certains bâtiments de haut standing. Ainsi, à Narbonne, au Clos de la Lombarde (Coupry, 1994), l'analyse met en évidence une terre provenant de Monte-Baldo ou de Chypre tout comme à Léro. Plus récemment, une boulette de pigment trouvée à Nîmes sur la fouille du parking Jean-Jaurès ${ }^{2}$ a également été identifiée comme étant de la céladonite. Sur le même site, trois décors au moins présentaient des fonds de ce riche vert souvent additionné de bleu égyptien ${ }^{3}$ (Boislève et al., 2011b). Deux d'entre eux accompagnent des sols à mosaïques polychromes, dont un pavement de près de $35 \mathrm{~m}^{2}$ représentant l'épisode mythologique de la mort de Penthée dans une pièce d'apparat d'une des plus luxueuses domus de ce quartier.

\section{La terre et l'enduit, une variété de pratiques}

Si la terre est, en matière de peinture, une source présence d'oxydes de fer (hématite et goethite de pigment très largement employée, elle entre aussi en compte comme matériau de construction lié à l'enduit ou constitutif de celui-ci. L'enduit de finition en terre augmenté d'un décor peint est désormais bien avéré pour les périodes préromaines (Barbet, 2008; Chazelles, 1995), même si les témoins, du fait de la fragilité de ce matériau, en demeurent épars et peu nombreux. Le plus ancien, qui reste une découverte unique, est signalé à Beaumont-surVesle (Marne) dans le cadre d'un diagnostic (Vanmoerkeke, Burnouf, 2006). D'importants 

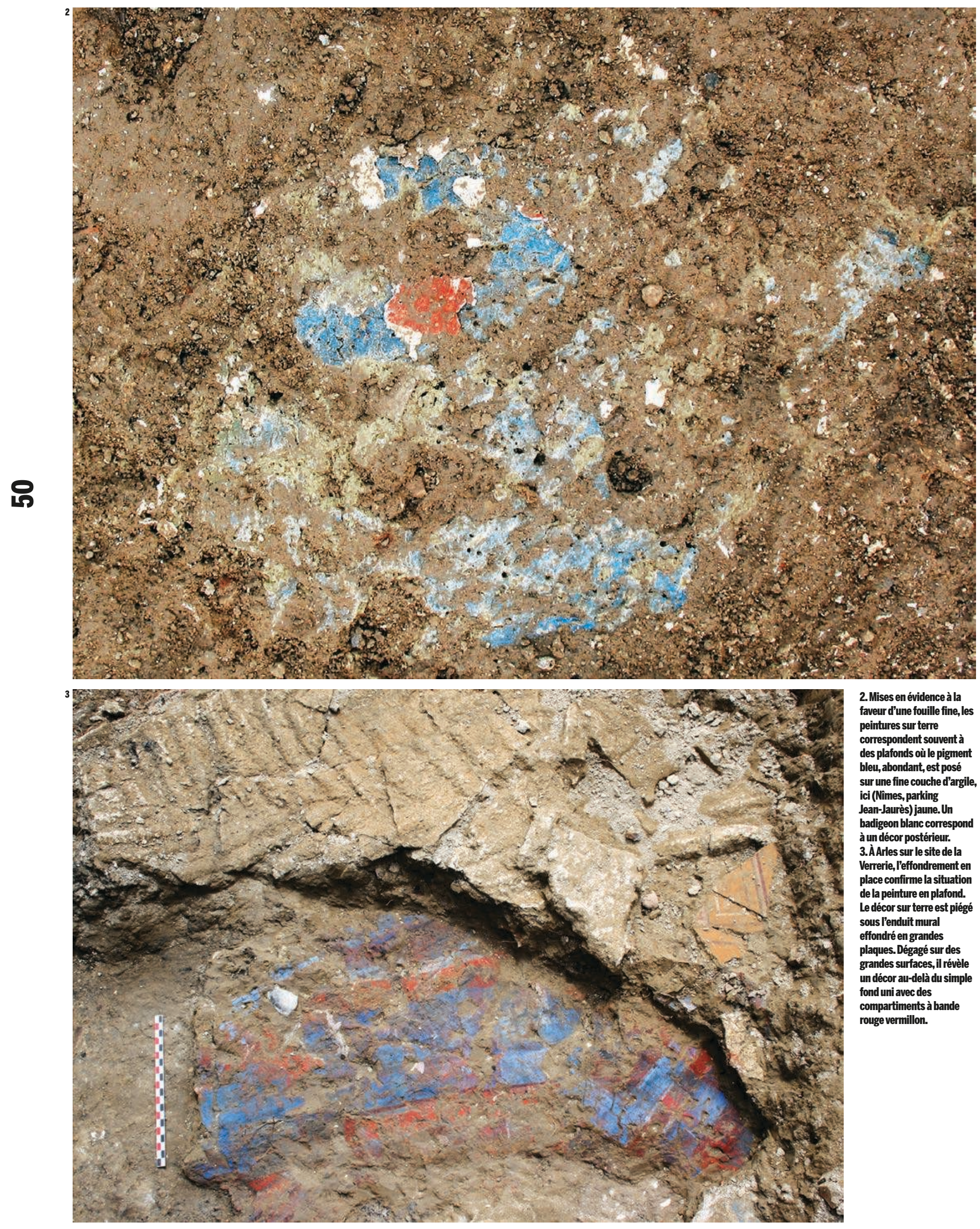

2. Mises en évidence à la faveur d'une foullle fine, les peintures sur terre correspondent souvent à des plafonds où le pigment bleu, abondant, est posé sur une fine couche d'argile, ici (Nîmes, parking Jean-Jaurès) jaune. Un badigeon blanc correspond à un décor postérieur. 3.ÀArles sur le site de la Verrerie,l'effondrement en place confirme la situation de la peinture en plafond. Le décor sur terre est piégé sous l'enduit mural effondré en grandes plaques. Dégagé sur des grandes surfaces, il révèle un décor au-delà du simple fond uni avec des compartiments à bande rouge vermillon. 
fragments de torchis rubéfiés conservent un décor à champs blancs et rouges daté de l'âge du Bronze. Les découvertes se font plus nombreuses pour la période couvrant l'âge du Fer, à Vix, Bourges, Lattes, Les Baux-de-Provence ou encore Nîmes parmi d'autres. Le décor n'est jamais parfaitement caractérisé faute de surfaces conservées suffisantes. En revanche, les analyses du support, notamment à Bourges (Cammas, 2007) ou à Vix (Allag, Coutelas, 2011) montrent l'emploi d'une argile ou d'un limon carbonaté mais surtout l'absence de chaux intentionnellement ajoutée dans le support. À l'inverse, une récente analyse de quelques fragments de La Tène ancienne, à Pasly (Aisne), traduit l'ajout volontaire de chaux à l'argile (Groetembril, 2014). L'enduit de terre est donc une pratique ancienne qui ne disparaît pas totalement avec l'apport des techniques romaines, même si elle est fortement supplantée par le mortier de chaux et la peinture à fresque. On observe ainsi depuis quelques années, et à la faveur d'une attention plus importante portée aux décors peints lors des fouilles, la présence récurrente de peintures sur terre [ill. 2]. Fragile, difficile à dégager et à prélever, ce mobilier archéologique reste délicat à appréhender et ne permet jamais, jusqu'à ce jour, de reconstitutions conséquentes des décors. Il apparaît toutefois que cette pratique est privilégiée, à la période romaine, pour les décors des plafonds. En effet, dans les quelques cas où il est incendié et où la terre est indurée, on perçoit au revers de l'enduit des empreintes de lattis ou de roseaux qui signent son accrochage en couvrement de pièce. Son association à des enduits muraux sur mortier, dans des effondrements en place, permet également de prouver sa situation en plafond comme à Arles ${ }^{4}$ [ill. 3] (Boislève, 2014), à Nîmes (Boislève et al., 2011a) ou à Die ${ }^{5}$ (Ronco, 2014 ; Boislève, Ronco, à paraître) par exemple. Dans tous les cas identifiables, il s'agit d'une terre fine recouvrant un support de roseaux ou de lattis de bois. La terre doit ici être appréciée pour sa plasticité qui permet d'épouser et de pénétrer le support irrégulier aménagé afin de garantir une bonne adhérence de l'enduit.

En surface, une mince couche d'argile assure généralement un épiderme qui permet un lissage fin, à l'image de la couche de chaux ou de poudre de marbre qu'on observe sur les enduits de mortier. Le pigment est appliqué directement sur cette couche de terre, loin donc de toute prise à fresque, ou sur un fin badigeon de chaux intermédiaire. Cette pratique, qui correspond à des décors où prédominent les fonds bleu égyptien, cohabite chronologiquement avec d'autres réalisés sur mortier. De même, on observe son usage du I ${ }^{\text {er }}$ siècle avant notre ère, comme à Arles sur le site de la Verrerie, et au moins jusqu'au début du $\mathrm{II}^{\mathrm{e}}$ siècle à Nîmes. Il ne s'agit donc pas d'une évolution technologique progressive où le mortier supplanterait la terre qui resterait employée un temps aux débuts de la conquête, mais bien d'une technique mise en œuvre pour ses qualités propres à certains contextes architecturaux ou à certains types de décors et notamment à l'application de certains pigments. Sans constituer la totalité du support de l'enduit, la terre intervient aussi fréquemment en couche de préparation pour la fixation des mortiers en plafond [ill. 4].

Plus inattendu est l'emploi de la terre en couche de finition sur un enduit de mortier, c'est-à-dire celle qui est la plus finement lissée afin de recevoir la peinture. Nous n'en connaissons à ce jour qu'un exemple en Gaule, découvert à Arles sur le site de la Verrerie [ill. 5] mais un cas similaire a été observé à Brescia en Italie. Bien que cette couche de terre fine et homogène ait engendré une très mauvaise conservation du décor, il apparaît que celui-ci est une nouvelle fois à dominante bleu égyptien, ce qui interroge sur un éventuel emploi privilégié de la terre en lien avec ce pigment. À ce stade, il est difficile de percevoir l'intérêt de cette technique qui, en l'absence de chaux dans la couche de finition, annule de fait les effets de fixation durable du pigment liés à la fresque. La terre peut-elle par exemple constituer un palliatif d'une poudre de marbre qu'il aurait été difficile de se procurer à un moment donné? Offre-t-elle des caractéristiques techniques qui permettent de mieux étaler un pigment bleu qui ne se dissout pas et conserve une granulosité importante?

C'est encore le mode de fixation de l'enduit sur une architecture de terre que l'on peut évoquer par l'analyse des revers du mortier. En effet, la pose de l'enduit nécessite son application sur une surface relativement rugueuse pour permettre son accrochage. Si les murs maçonnés en moellons ou en brique présentent les aspérités nécessaires, ceux en terre et en bois offrent une surface trop lisse qui implique une préparation de la paroi pour recevoir l'enduit. Plusieurs techniques sont observées même si les incisions rectilignes disposées en chevrons sont les plus fréquentes [ill. 6]. Les rangs de lignes brisées, plus ou moins régulières, peuvent être disposés aussi bien verticalement qu'horizontalement, les deux sens cohabitant même parfois sur une seule paroi. Certains revers aux traces particulièrement régulières et disposées en bandes de même largeur ont pu faire penser à l'emploi de rouleaux, sortes de molettes gravées qui laisseraient sur la terre humide les reliefs permettant l'accrochage de l'enduit. L'hypothèse a notamment été avancée à Paris où plusieurs décors livrent des empreintes en forme de petits losanges inscrits de dimensions standardisées qui évoquent un estampage, mais développé ici sur une couche de mortier et non sur la terre (Eristov, Robin, 2011). On note aussi des traces en sinusoïdes, obtenues à l'aide d'une sorte de peigne dentelé et non pas de la main comme le suggèrent encore quelques publications. Le piquetage, généralement réservé à la pose d'un nouvel enduit sur un précédent, peut également être observé sur un mur en terre, comme à Nîmes sur l'un des décors du parking Jean-Jaurès. Il laisse au revers de l'enduit de petites proéminences caractéristiques de ce procédé. 

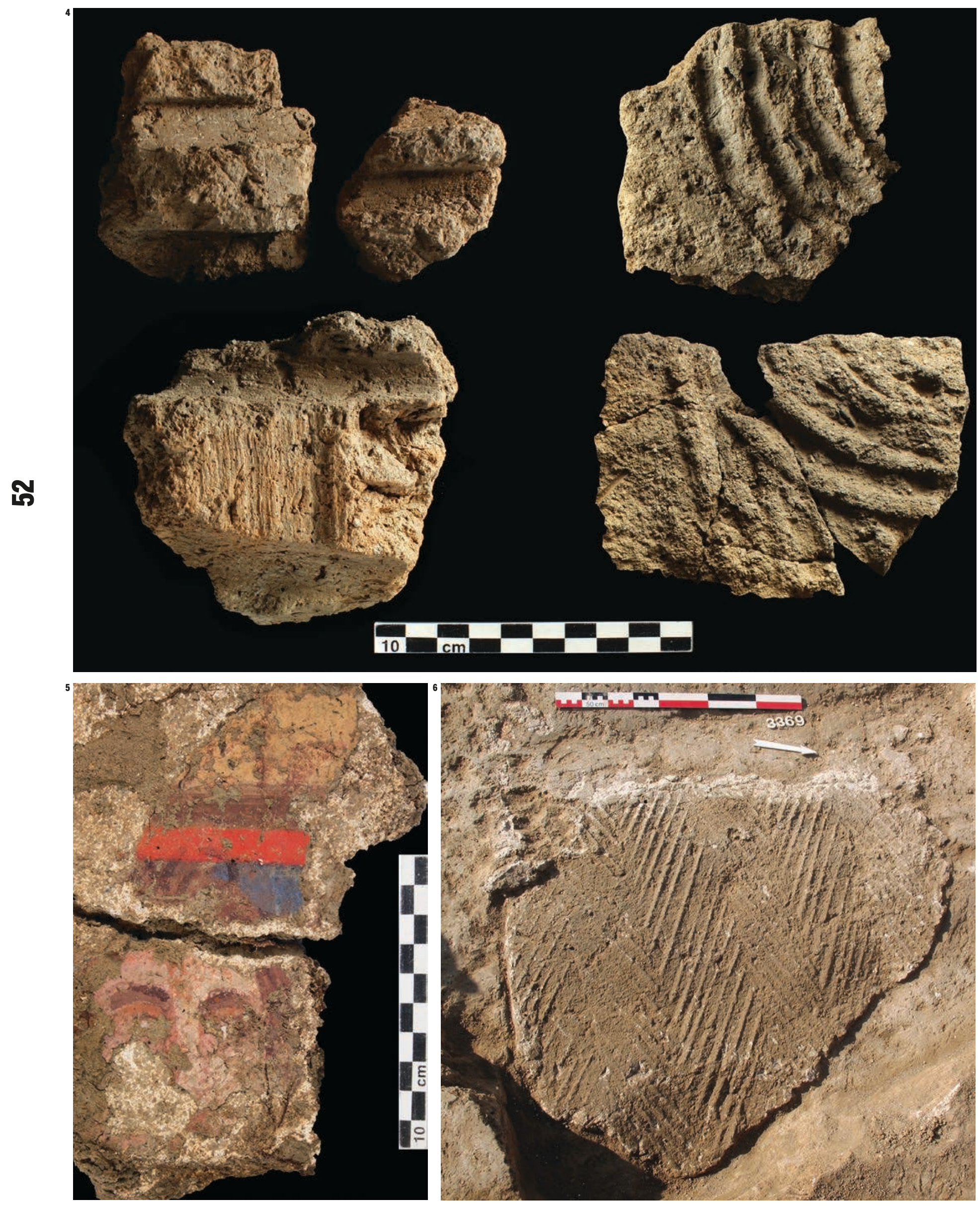
4.L'incendie des pièces de bois des plafonds, poutres et solives a cuit la terre qui en a conservé l'empreinte. Sur ces fragments de plafond, à Mané-Véchen (Morbihan), un enduit de terre était appliqué sur un clayonnage de baguettes et de lattes fixées aux solives (à gauche). Sa surface était ensuite striée au peigne pour permettre l'accrochage des couches de mortier (à droite). 5. Sur cet enduit du site de la Verrerie à Arles, le support de mortier est complété par une couche d'épiderme constituée d'une argile grise sur laquelle est appliqué le pigment. La présence d'un décor figuré sur un fond bleu cerné de vermillon indique qu'il ne s'agit pas d'une pratique à

l'économie et qu'il faut plutôt chercher dans son emploi une qualité technique.

6. Au revers de l'enduit, les stries en chevrons marquent le négatif des incisions pratiquées sur un mur de terre pour garantir l'adhérence du mortier. Le sens des chevrons et la régularité des incisions sont variables. Ici à Nîmes, parking Jean-Jaurès, les traces sont particulièrement régulières.
6. Fouille du Parc

d'activité menée en

2007 sous la direction

de Gérard Bataille, Inrap.

7. Fouille de la rue

Romaine, menée en 2013 sous la direction de

Stéphane Venault, Inrap.

8. Fouille menée en

2008 sous la direction

de P. Cayn, Inrap.
L'enduit, révélateur de l'architecture en terre

Au-delà de l'observation des techniques d'emploi de la terre mises en évidence dans la réalisation de l'enduit, c'est l'apport en termes de restitution architecturale qui livre le plus d'informations sur l'usage de ce matériau. Couvrant le plus souvent toute l'élévation d'une pièce, des murs au plafond, l'enduit constitue un véritable moulage des volumes de ces espaces, comme l'épiderme d'une architecture. Matière à la plasticité plus ou moins prononcée, l'enduit, qu'il soit de mortier ou de terre, épouse son support avec souvent suffisamment de finesse pour en conserver l'empreinte. Dès lors, l'observation du revers des peintures apporte une série d'indices permettant de caractériser la nature du mode de construction adopté pour les murs et les plafonds.

Dans le cas d'une construction en terre, l'élévation a le plus souvent disparu par le pourrissement des matériaux périssables qui en constituent la structure de bois, par la fonte de la terre sous l'action de l'eau ou encore par l'évacuation volontaire des matériaux liée à la récupération des pierres à la base d'un mur. L'enduit, plus résistant et moins intéressant en termes de récupération de matériaux, devient donc un indice précieux pour identifier une construction à base de terre. Si les traces d'accrochage au revers révèlent la nature du matériau employé, en l'occurrence les chevrons ou sinusoïdes pour la terre, le type exact de mise en œuvre reste souvent difficile voire impossible à préciser. En effet, la surface d'un mur en terre banchée comme celle d'une paroi en bauge ou en adobe ne présente pas de traces suffisamment explicites pour différencier ces trois cas. Les incisions destinées à assurer l'adhérence de l'enduit et pratiquées sur ces murs ou sur un enduit de terre intermédiaire sont les mêmes.

Lorsqu'il s'agit de constructions en torchis sur armature de bois et clayonnage, l'incendie des structures permet dès lors, par cuisson de la terre, de conserver toutes les couches en lien avec l'enduit et d'y lire l'empreinte des éléments périssables. Ainsi, à Eckbolsheim ${ }^{6}$ (Bataille, 2014), l'analyse des peintures (Brunet-Gaston, 2011) met en évidence toute la structure à colombages avec hourdis de torchis sur clayonnage qui constituaient les parois de cette pièce semi-enterrée d'une villa. La lecture, en parallèle, du décor et des revers permet de replacer les éléments et de définir précisément le mode de construction. Des dés en grès, conservés en place dans la pièce, constituent l'ancrage de poteaux de bois dont l'enduit conserve le profil circulaire et le diamètre s'établissant à $20 \mathrm{~cm}$. Entre ceux-ci, la zone inférieure présente une sablière basse surmontée d'un renfort en croix de SaintAndré supportant un bardage à clins. Au-dessus, des panneaux à clayonnage d'éclisses servent de support au torchis. La surface de celui-ci est striée au peigne pour accueillir un torchis plus fin en couche préparatoire de l'enduit.

Le même type d'observations est valableà Entrainssur-Nohain ${ }^{7}$ où l'enduit est encore adhérent au support de terre rubéfiée qui conserve les empreintes de la structure de bois constitutive des parois [ill. 7]. Celle-ci présente une armature de montants et de traverses de bois à face plate entre lesquels prennent place des clayonnages à baguettes de bois ou d'osier de sections circulaires de 1 à $2 \mathrm{~cm}$ de diamètre. La disposition des clayonnages, parallèlement ou perpendiculairement aux pièces de bois à face plate (leur probable section carrée ne peut être assurément restituée), autorise à restituer une structure à assemblage de poutres verticales et horizontales. Le clayonnage se situe en retrait par rapport à la surface des montants de bois. L'ensemble est recouvert de deux couches de torchis et trois couches de mortier. La première couche de terre, épaisse d'environ $2 \mathrm{~cm}$, permet de rattraper le décalage entre clayonnage et montants de bois. Elle est striée au peigne, en mouvements sinusoïdaux, pour faciliter l'adhérence d'une seconde couche de terre, d'une épaisseur équivalente, qui offre une même surface de terre sur l'ensemble de la paroi, recouvrant donc aussi les montants de bois. La surface est striée selon le même procédé mais cette fois pour l'accrochage du mortier constituant la couche de préparation de l'enduit.

Le mur en terre et bois peut être directement implanté au sol ou posé sur lui comme pour des petites cloisons internes telles que nous pouvons en restituer dans une pièce de la grande domus du quartier Clérisseau à Nîmes ${ }^{8}$ (Cayn, 2011 ; Boislève, Cayn, 2012 ; Boislève, Cayn, 2013). Le sol de béton lissé a été posé sur une large surface ensuite subdivisée en trois pièces par l'ajout de cloisons de terre non fondées. Mais le plus souvent, pour pallier les risques de dégradation liée aux remontées d'humidité, la paroi de terre se développe au-dessus d'un soubassement maçonné ou mur-bahut. La reconstitution de grandes plaques de peinture permet souvent de lire au revers de l'enduit les empreintes différentes entre soubassement maçonné et élévation de terre. Le passage de l'un à l'autre peut être observé et, lorsqu'il n'est pas conservé, la hauteur du mur-bahut restituée. Elle peut être assez variable selon les régions mais aussi selon la place de la paroi, étant réduite à un simple solin de pierres d'une ou deux assises dans le cas d'une petite cloison interne. À Nîmes, la fouille du parking Jean-Jaurès a permis l'exploration partielle de huit îlots d'habitation où l'architecture en adobe sur soubassement maçonné de moellons est le mode de construction prédominant. Les décors retrouvés effondrés en place dans de nombreux cas permettent de mieux appréhender l'élévation des bâtiments. On observe ainsi des pratiques assez normées. Le mur-bahut atteint en général une hauteur de 40 à $45 \mathrm{~cm}$, représentant trois à cinq assises et dépassant donc largement le pied de hauteur minimale prescrite par Caton (Caton, 1975, XVII, 14, 4) pour le soubassement d'un mur de terre. De même, leur largeur varie selon qu'il s'agit là encore de murs porteurs, en façade notamment, ou de cloisons de division interne et de refends. Il apparaît ainsi que les murs porteurs présentent une largeur 


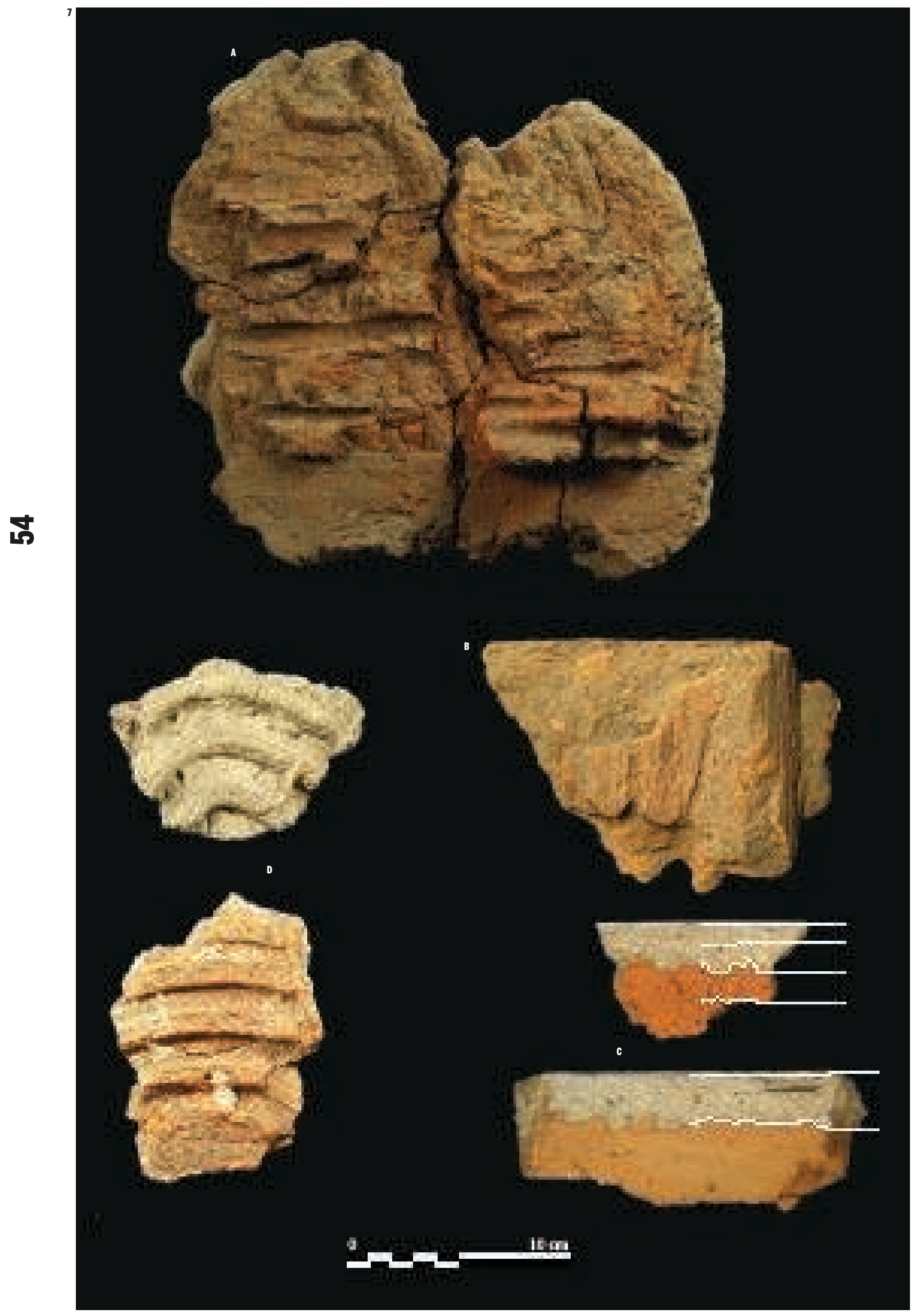

7.Entrains-sur-Nohain, rue Romaine. Les enduits

brûlés conservent un

support de terre

permettant de rétablir une

paroi à pans de bois et

remplissage de clayonnage

(A). Quelques fragments

prouvent la disposition à

angle droit des poutres du

pan de bois (B). Le torchis

est passé en deux couches

(C), chacune étant striée

au peigne pour

l'accrochage de la couche

de terre suivante ou de

l'enduit (D).

Itri 
de 40 à $46 \mathrm{~cm}$, soit à peu près une coudée, alors que les cloisons peuvent n'être larges que de 15 à $16 \mathrm{~cm}$. L'enduit permet parfois d'observer un rattrapage de niveau entre le soubassement et l'élévation en terre. Ainsi, dans une pièce de l'îlot $\mathrm{G}$ (Boislève et al., 2011a), l'enduit marque un angle saillant assez prononcé entre zone inférieure et zone médiane du décor. La surface de cette dernière se trouve alors en retrait de 2 à $3 \mathrm{~cm}$ par rapport à celle de la zone inférieure. Le revers de l'enduit indique que ce décalage s'opère au passage entre élévation maçonnée et construction en terre. Le soubassement de pierre étant manifestement plus large $(25 \mathrm{~cm})$ que les modules de briques d'adobes (estimés à $20 \mathrm{~cm}$ ), l'artisan peintre a dû intégrer ce décrochement au décor par un angle soigné évitant d'avoir à poser en zone médiane une surface trop épaisse de mortier.

Dans plusieurs pièces de ce même site, l'effondrement en place des décors a permis une reconstitution suffisamment importante pour connaître la hauteur précise sous plafond et prouver même l'existence d'un étage [ill. 8]. Il devient dès lors possible de raisonner sur la portée des murs de terre en fonction de leur largeur et de vérifier ainsi les écrits antiques. En effet, Vitruve note qu'il convient de faire un mur d'au moins un pied et demi d'épaisseur pour soutenir un étage (Vitruve, 2005, II, 8). À Nîmes, plusieurs pièces de l'îlot $C$ autorisent la restitution d'un étage, prouvé par l'effondrement en place et simultané de deux décors. Au rez-de-chaussée, la recomposition des peintures rétablit une hauteur de l'ordre de $2 \mathrm{~m}$ confirmée par la présence de graffitis jusqu'à $9 \mathrm{~cm}$ sous la bande marquant le sommet de la paroi. Cette faible hauteur du rez-de-chaussée, qui s'explique justement par la présence d'un étage, se retrouve dans des proportions équivalentes dans un autre bâtiment du même site, appartenant à l'îlot E. Dans une aile de la domus E8, trois pièces en enfilade livrent encore des décors effondrés en place ainsi que, dans un cas, le sol de béton de l'étage. Le remontage complet de la hauteur du décor, dans les deux pièces, indique une amplitude sous plafond de 2,01 et 2,07 m. Dans le cas de ces constructions en adobe, la présence d'un étage impose donc une pièce de rez-de-chaussée de hauteur très limitée, ce qui n'en fait pourtant pas des pièces secondaires si on en juge par la présence du décor simple mais d'assez bonne qualité qui y est développé.

Dans ces deux cas, les murs de façade présentent une épaisseur de 4.0 à $45 \mathrm{~cm}$ soit tout juste le pied et demi indiqué par Vitruve. Le décor de l'étage présentant le même rythme de panneaux qu'au rez-de-chaussée, il faut encore envisager des pièces du même ordre de hauteur, soit environ $2 \mathrm{~m}$, hors pignon. Un mur d'adobe de 1,5 pieds de largeur peut donc offrir une élévation de plus de $4 \mathrm{~m}$. Ce constat se vérifie encore à Nîmes, sur le site de la percée Clérisseau où une petite cloison interne était effondrée d'un seul tenant sur le sol de béton. La recomposition du décor rétablit une paroi de 4,80 m de hauteur. Si la cloison de seulement $16 \mathrm{~cm}$ d'épaisseur est entièrement en adobe, les murs porteurs de la pièce présentent un soubassement de pierres surmonté d'une architecture de terre comme le révèlent les chevrons au revers de l'enduit. La largeur du mur est de l'ordre de $50 \mathrm{~cm}$. Le rapport généralement admis d'une élévation pouvant atteindre huit fois la largeur du mur se vérifie donc.

Enfin, il faut noter le rôle de l'enduit sur une paroi. Il constitue aussi un mode de protection et de finition du mur, particulièrement lorsque celui-ci est en terre. Laissé blanc, comme on l'observe notamment sur des enduits de façade, il assure une couche protectrice à la fois du mur en terre et des structures en matériaux périssables qui peuvent le constituer. Mais il joue avant tout un rôle décoratif et permet, selon la qualité du décor développé, l'embellissement d'une architecture. Dans les premiers développements de la peinture romaine, sur les décors de $\mathrm{I}^{\mathrm{er}}$ et de $\mathrm{II}^{\mathrm{e}}$ style, on note d'ailleurs une volonté d'imiter, en stuc et en peinture, une architecture de grande qualité avec des blocs de grand appareil ou des imitations de placages de marbre. Cet enduit masque parfois une architecture jugée plus modeste, y compris en terre, et lui donne l'aspect d'un tout autre standing.

Un rapide tour d'horizon des décors peints connus en Gaule fait néanmoins apparaître d'emblée l'absence de corrélation entre statut social de l'habitat et construction en terre. C'est bien le décor, selon la complexité des compositions, le raffinement des motifs ou même le coût des pigments employés, et les aménagements de confort qui font la richesse des pièces. Ainsi, au $\mathrm{I}^{\mathrm{er}}$ siècle avant notre ère, les remarquables décors de $\mathrm{II}^{\mathrm{e}}$ style, par exemple à Glanum ou à Arles, qui sont l'œuvre de peintres qualifiés venus vraisemblablement d'Italie et usant largement du cinabre, un pigment parmi les plus coûteux, revêtent des murs en terre sur soubassement de pierre. Et le constat ne se dément pas au fil de l'époque romaine comme le prouvent la riche peinture au génie ailée du Clos de la Lombarde à Narbonne et, à Nîmes, celle à l'Apollon citharède sur fond céladon, développées au $\mathrm{II}^{\mathrm{e}}$ siècle de notre ère, toujours sur des parois en adobe.

Le dialogue entre terre et enduit est donc étroit, celle-ci servant tantôt de support à l'autre, de matériau constitutif ou même de peinture à proprement parler. L'archéologie du décor parvient aujourd'hui plus précisément à identifier l'emploi de la terre au sein des enduits, sans parvenir encore à bien définir les raisons techniques de tels choix, ce que l'archéologie expérimentale permettra sans doute de mieux cerner. Les analyses physico-chimiques documentent de plus en plus précisément la mise en ouvre de ce matériau et devraient permettre à terme de mettre en évidence des pratiques locales ou de certains ateliers. Mais, d'ores et déjà, la recomposition des enduits peints offre la possibilité de rétablir les volumes et l'élévation des bâtiments tout en précisant leur mode de construction. Ils permettent donc de lire une architecture en terre, fréquente et par nature mal conservée, parfois dans son intégralité. 


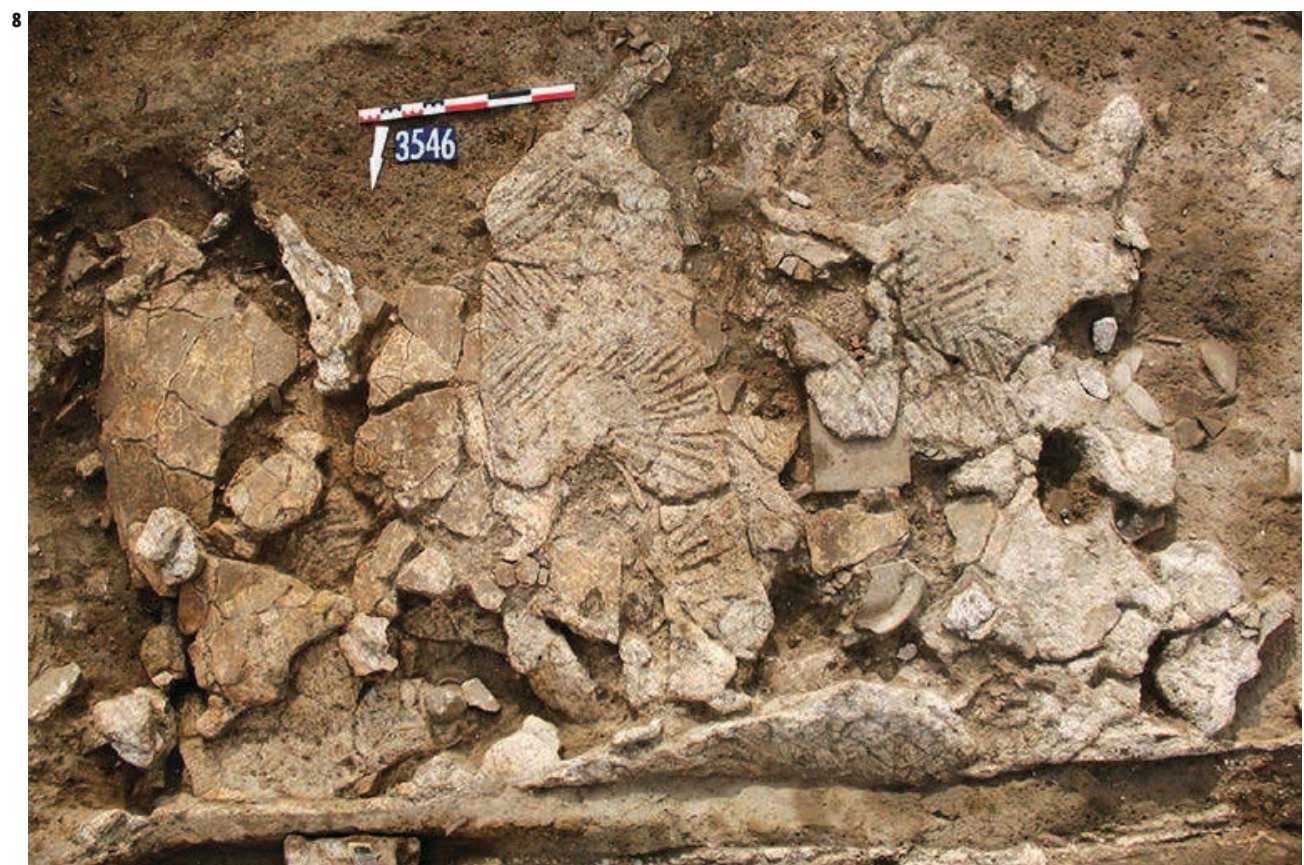

i̊

8. Nîmes parking JeanJaurès, l'effondrement en place des décors et parfois même d'un sol de béton a permis de prouver l'existence d'un étage. Le revers des peintures, aux traces d'accrochag en chevrons, prouve une

\section{construction en terre} aussi bien pour les murs porteurs que pour les petites cloisons de subdivision des pièces et la recomposition du déco livre la hauteur précise de la pièce au rez-dechaussée.

\section{完}

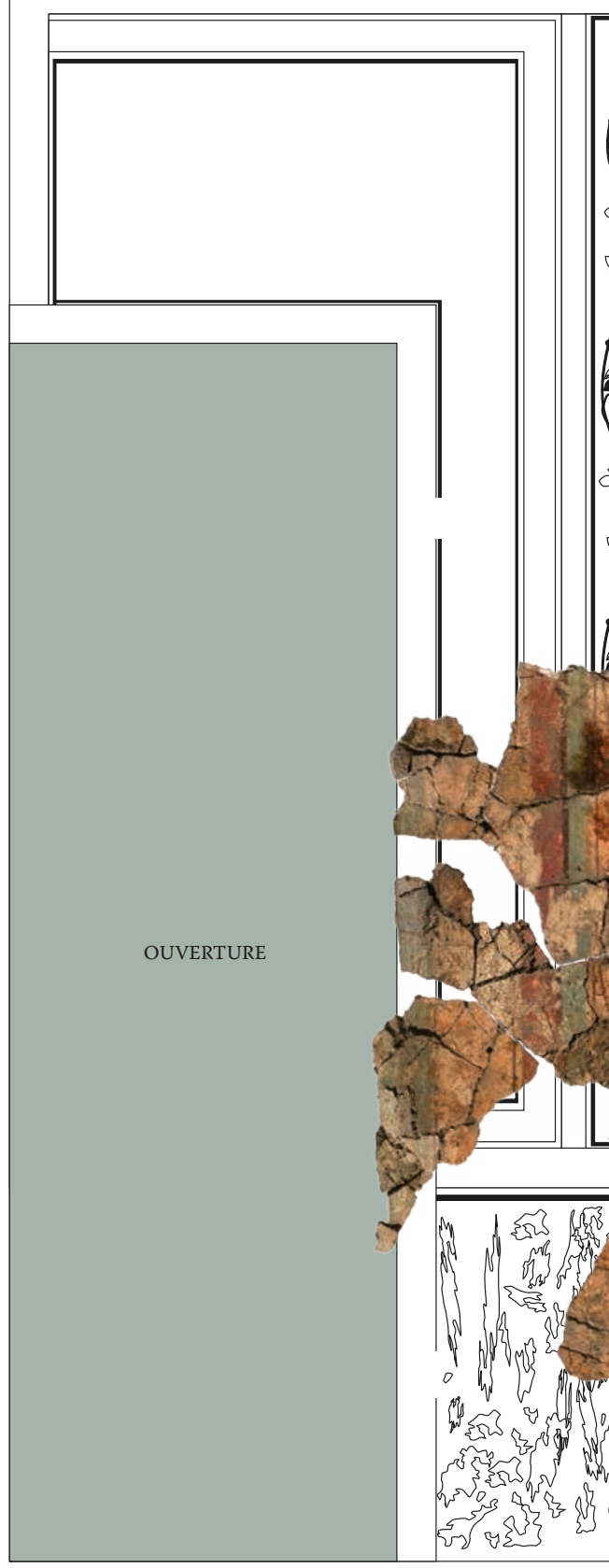

3 vol. $(212,459$ et $697 \mathrm{p}$.)

Boislève J., COllab. Rothé M.-P., Genot A., 2014, « Nouvelles découvertes sur le site de la Verrerie à Arles: de remarquables peintures murales de deuxième style pompéien ", in CAPUS P., Dardenay A. (CoORd.), L'empire de la couleur, de Pompéi au sud des Gaules, Toulouse, Musée SaintRaymond, p. I-IV.

Boislève J., Breuil J.-Y., CAYN P., Houix B., Vauxion O., 2011a, « Architecture et décor d'une domus dans le quartier sud-ouest de Nîmes durant le HautEmpire : la fouille du parking Jean-Jaurès, îlot G », in BALMELLE et al. 2011, p. 49-66.

Boislève J., Breuil J.-Y., Houix B., Vauxion O., 2011b, «Mosaïques et peintures d'un quartier de Nemausus : la fouille du parking Jean-Jaurès à Nîmes (Gard) », in BALMELle et al. 2011, p. 33-48.

Boislève J., CAYN P., 2012, « Peintures et mosaïques d'époque romaine : les décors du quartier Clérisseau à Nîmes (Gard) », in FuCHS M., MONIER F. (DIR.), Les enduits peints en Gaule romaine: approches croisées, Actes du $23^{e}$ séminaire de l'Association française pour la peinture murale antique, Paris, 13-14 novembre 2009, Dijon, Société archéologique de l'Est (coll. Suppl. à la RAE, 31), p. 39-52.

Boislève J., Cayn P., collab. Tendron G., Fuchs M., 2013, «Les fouilles du quartier Clérisseau à Nîmes : analyse des décors d'une vaste domus », Revue archéologique de Narbonnaise, 45, p. 257-291.

Boislève J., Dardenay A., Monier F., 2014, Peintures et stucs d'époque romaine : révéler l'architecture par létude du décor, Actes du $26^{\mathrm{e}}$ colloque de l'AFPMA, Strasbourg, 16 et 17 novembre 2012, Bordeaux Ausonius (Pictor, collection de l'AFPMA, 3), 345 p. BoIslève J., Ronco C., à paraître, « Pan, Bacchus et le sphinx, une peinture murale d'époque romaine découverte à Die (Drôme) », in BoISLÈve J., Dardenay A., Monier F., Actes du $27^{\circ}$ colloque de l'AFPMA, Toulouse, 21-22 novembre 2014, Bordeaux, Ausonius (Pictor, collection de l'AFPMA, 5)

Brunet-Gaston V., Collab. Monier F., 2011, «Une pièce semi-enterrée à Eckbolsheim (Bas-Rhin) et sa décoration peinte », in BALMELLE et al. 2011, p. 321-328. 


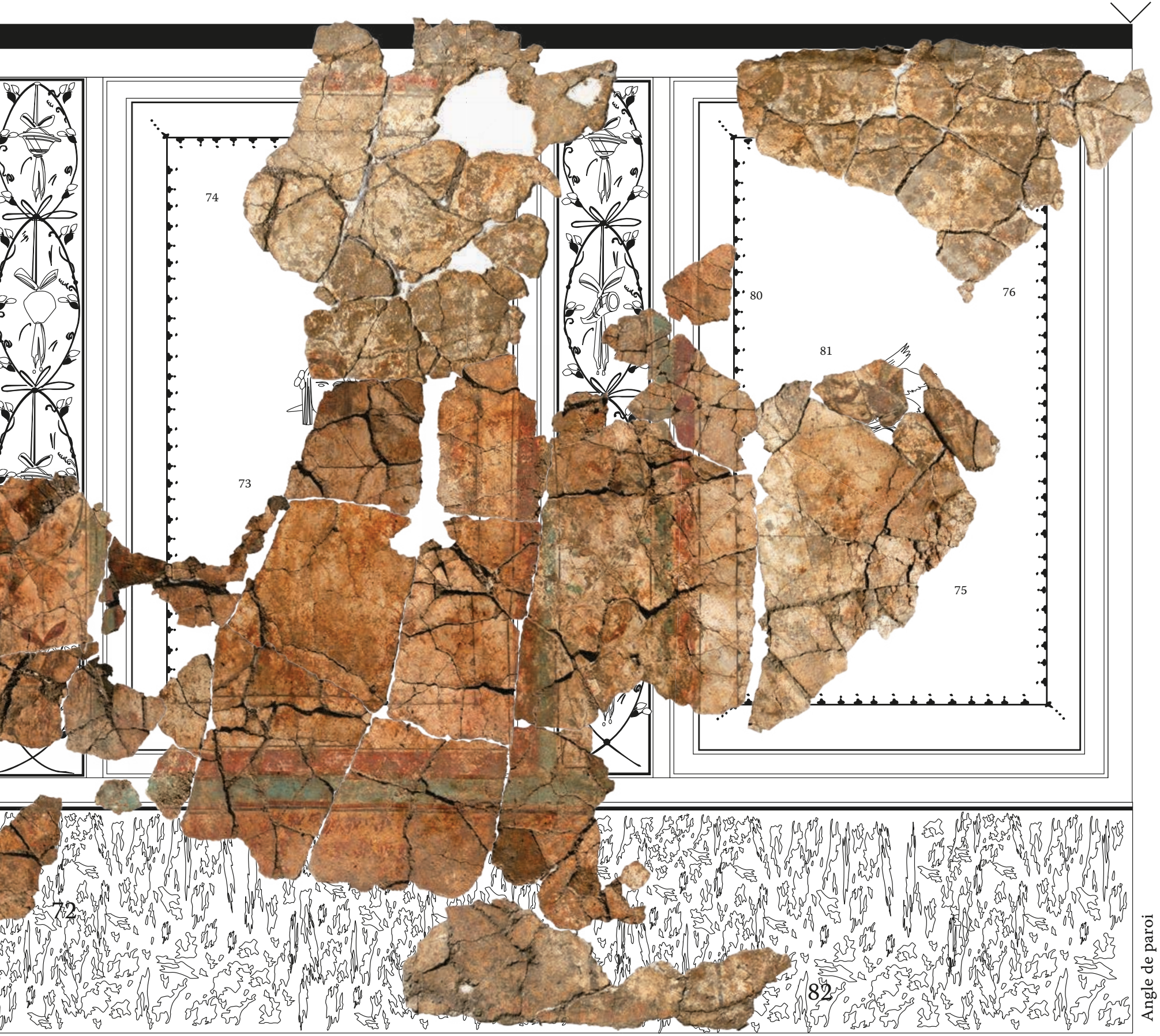

Cammas C., 2007, « Approche micromorphologique du traitement de surface des murs sur les sites de Littré et de l'hôtel-Dieu », in AUgIER L., Buchsenschutz O., RALSTON I., Un complexe princier de lâge du Fer, l'habitat du promontoire de Bourges (Cher), $V I^{e}-I V^{e}$ siècle av. J.-C., Bourges, Bourges plus service d'archéologie préventive (coll. Suppl. à la RACF, 32 / Bituriga Monographie, 3), p. 135-147.

CAYN P. (DIR.), 2011, Les 2000 ans d'histoire du quartier Clérisseau: de l'urbanisation antique à nos jours (Languedoc, Gard, Nîmes, Clérisseau 5), rapport d'opération, Inrap-SRA Languedoc-Roussillon, 4 vol. (313, 180, 360 p.).

Chazelles C.-A DE., 1995, « La maison préromaine et son décor en Gaule méridionale », Revue archéologique de Picardie, 10-1, p. 35-40.

Coupry C., 1994, « Annexe. Analyse physico-chimique des pigments de la Maison III ", Revue archéologique de Narbonnaise, 27-28, p. 242-251.
DelamARE F., 1987, « Les terres vertes et leur utilisation en peinture murale romaine ", in Delamare F.,

HaCKENS T, Helly B. (ÉD.), Datation-caractérisation des peintures pariétales et murales, Ravello, Centre universitaire européen pour les biens culturels (coll. PACT, 17), p. 345-373

Eristov H., RobIN S., 2011, « Les décors peints de Lutèce : bilan des découvertes », in BALMELLE et al. 2011, p. 131-149.

Foucault A., RaOult J.-F., 2005, Dictionnaire de géologie, $6^{e}$ édition, Paris, Dunod, 382 p.

Groetembril S., 2014, « Pasly, enduits de La Tène ancienne ", in BOISLÈVE et al., p. 301-308.

Ronco C. (DIR.), 2014, Die (Drôme), Place de la Cathédrale - place du Marché/ Aménagement des places, rapport d'opération, Inrap-SRA Rhône-Alpes, 3 vol. (197, 192, 370 p.)

VANMOERKEKE J. BURNOUF J. (COORD.), 2006, Centmille an sous les rails: archéologie de la ligne à grande vitesse est européenne, Paris, Inrap / Somogy éditions d'art, 135 p. 